\title{
Diagnostic Ureteroscopy in CT Urography- Diagnosed Upper Tract Urothelial Carcinoma: Delay in Definitive Treatment and Increased Intravesical Recurrence
}

\author{
Hadi SHSM ${ }^{1}$, Elizabeth Bright ${ }^{1}$, Mark Mantle ${ }^{1}$, Nicholas Munro ${ }^{1}$, Omar Fahmy ${ }^{2}$ \\ 1. Urology, Cornwall Royal Hospital, Truro, GBR 2. Urology, Universiti Putra Malaysia, Serdang, MYS
}

Corresponding author: Hadi SHSM, hadi.mohsin@nhs.net

\section{Abstract \\ Purpose}

To investigate the effect of diagnostic ureteroscopy (URS) on the delay to surgical treatment of upper tract urothelial carcinoma (UTUC) detected by imaging and the risk of intravesical recurrence.

\section{Materials and methods}

We undertook a retrospective case-note analysis of all patients who underwent radical nephroureterectomy (NUU) from November 2012 to July 2019. We identified those who underwent diagnostic ureteroscopy prior to NUU as Group 1 and those who did not undergo diagnostic URS as Group 2. Perioperative and pathological parameters were compared between both groups. Kaplan-Meier and Log-Rank analyses were used to compare delay to NUU and the intravesical recurrence (IVR) free survival. Cox regression models were employed to analyze the risk factors of intravesical recurrence.

\section{Results}

Out of 69 patients with a mean age of 71.3 years and a mean follow-up of 48.5 months, 49 (71\%) underwent URS while 20 (29\%) did not. The mean time between the computerized tomography urography (CTU) and surgery was 86 days with URS and 59 days in the control groups $(\mathrm{p}=0.007)$. Intravesical recurrence in year one postoperatively was $28.2 \%$ in the URS group vs $5.9 \%$ in the control group ( $\mathrm{p}=0.04)$. The Kaplan-Meier curve showed improved, yet insignificant, IVR-free survival for the control group (Log-Rank $\mathrm{p}$-value $=0.21$ ). In multivariate Cox regression analysis, concomitant bladder carcinoma was an independent risk factor for IVR (HR, 15.01; 95\%CI, 3.311 - 68.07; $\mathrm{p}=0.0004$ ). Intravesical mitomycin-c was a protective factor (HR 0.154; 95\%CI $0.025-0.922 ; \mathrm{p}=0.040)$.

\section{Conclusion}

Review began 06/05/2021 Review ended 06/12/2021 Published 06/20/2021

\section{(๑) Copyright 2021}

SHSM et al. This is an open access article distributed under the terms of the Creative Commons Attribution License CC-BY 4.0., which permits unrestricted use, distribution, and reproduction in any medium, provided the original author and source are credited.
In our retrospective single-unit study, diagnostic ureteroscopy for CTU-detected upper tract urothelial carcinoma delayed definitive surgical treatment. Furthermore, it was associated with a significantly increased risk of early intravesical recurrence. URS can provide useful information and reassurance prior to major surgery but must be used with caution in light of these findings.

Categories: Urology, Oncology

Keywords: upper tract urothelial carcinoma, ureteroscopy, intravesical recurrence, nephroureterectomy, bladder cancer

\section{Introduction}

Upper tract urothelial carcinoma (UTUC) is a rare malignancy, constituting only $5 \%-10 \%$ of all urothelial carcinomas [1]. However, more than half of patients have an invasive disease at presentation [2]. Radical nephroureterectomy (RNU) is the gold standard treatment for patients with a high risk of metastatic disease, who represent the majority of the patients [3]. Techniques to determine disease stage and depth of invasion at presentation are limited. Computerized tomography urography (CTU) is currently the most accurate imaging modality to diagnose UTUC, with a sensitivity and specificity of $92 \%$ and $95 \%$, respectively [4]. Diagnostic ureteroscopy (URS) can be performed to resolve inconclusive CTU findings [3]; however, many urologists perform URS as a confirmatory tool for UTUC that is clearly diagnosed on CTU [5].

Diagnostic URS prior to NUU has both benefits and risks to the patient. One benefit of URS is the possibility to perform a biopsy and obtain histopathological confirmation of UTUC, as well as visually identify a presumed tumor. It is relatively unusual to submit patients to major cancer surgery without visual or histological confirmation of the diagnosis, i.e. to rely on the CTU alone. However, URS has the potential to increase time delay to RNU and delay definitive treatment and thus adversely affect the overall outcome [6]. 
A delay in definitive surgery from diagnosis is thought to be a risk factor for adverse outcomes; however, the threshold of significant delay is controversial and has been proposed to range from 30 to 90 days [3]. Additionally, It has been proposed that manipulation of the tumor during URS might increase the risk of intravesical recurrence (IVR) of urothelial cancer in the future $[5,7]$.

In our unit, we submit a proportion of those with putative UTUC to URS after individual case reviews at our multidisciplinary meeting (MDT). We wanted to assess the risks and benefits of doing so and better advise our patients for the future. Specifically, this study was undertaken to investigate the potential delay of diagnostic URS on the definitive surgical treatment for patients with UTUC detected by CTU. In addition, we assessed whether performing diagnostic URS in this situation increased IVR.

\section{Materials And Methods Study population}

After obtaining ethical committee approval, we undertook a retrospective analysis of all patients receiving RNU for UTUC at one institution between November 2012 and July 2019. Patients with normal findings on CTU were excluded from the analysis.

\section{Management}

All patients had either open or laparoscopic RNU with excision of the bladder cuff. Diagnostically rigid or flexible URS with or without biopsy was performed in selected patients after discussion at a multidisciplinary cancer meeting (MDT). Diagnostic cystoscopy was performed routinely for all patients as part of the diagnostic protocol. Postoperative intravesical mitomycin-c or systemic adjuvant chemotherapy were given on a case-by-case basis after ratification at the MDT.

\section{Follow-up}

All patients underwent radiological surveillance and regular cystoscopic examination post-RNU according to the European Association of Urology (EAU) Guidelines [4]. Both the development of metastatic disease and death due to UTUC were recorded with reference to duration since RNU.

\section{Data analysis}

We retrospectively reviewed clinical data and recorded the patient's characteristics, pathological features of the UTUC, the time interval between the CTU and the date of RNU. Follow-up and survival data were recovered.

Patients who underwent diagnostic URS were assigned as the study group. Patients who did not undergo URS were assigned as the control group. The primary outcomes of this analysis were: a) Impact of diagnostic URS on delay to surgery and b) Impact on intravesical recurrence incidence.

\section{Statistical analysis}

For this study, statistical analysis was performed using the JMP V.15.2.1 software (SAS Inc., Cary, NC). All data were presented as median and range for continuous variables and as absolute numbers with percentages for categorical variables. The Mann-Whitney U and Pearson $\mathrm{x} 2$ tests were employed to compare the continuous and categorical variables, respectively. Kaplan Meier curves were used to present the survival data with the log-rank test for comparison. Univariate and multivariate Cox regression analyses were conducted to assess the risk factors of intravesical recurrence. A p-value of $<0.05$ was regarded as significant.

\section{Results}

We identified 70 patients who underwent RNU. One patient was excluded from the study with normal findings on CTU and of the remaining 69 patients, 49 (71\%) underwent diagnostic URS. The remaining 20 patients (29\%) were assigned as a control group. The patient's perioperative characteristics and the comparison between the two groups are presented in Table 1 . There was no significant difference between both groups, except in gender ( $71 \%$ male and $29 \%$ female vs $45 \%$ and $55 \%$, respectively, $\mathrm{p}=0.04$ ) and in adjuvant chemotherapy administration ( $8.3 \%$ in the URS group vs $31.6 \%$ in the control group, $\mathrm{p}=0.02$ ). 


\section{Cureus}

\begin{tabular}{|c|c|c|c|c|}
\hline Variable & All patients $(n=69)$ & URS group(n=49) & Non-URS group $(n=20)$ & P-value \\
\hline Age & $71.3 \pm 8.4$ & $71 \pm 8$ & $72 \pm 10$ & 0.94 \\
\hline Male & 25 & $35(71 \%)$ & $9(45 \%)$ & \multirow{2}{*}{$0.04^{*}$} \\
\hline Female & 25 & $14(29 \%)$ & $11(55 \%)$ & \\
\hline Follow-up & $48.5 \pm 25$ & $52 \pm 24$ & $36 \pm 23$ & 0.10 \\
\hline Laparoscopic & 63 & $46(94 \%)$ & 17 (85\%) & \multirow{2}{*}{0.25} \\
\hline Open & 6 & $3(6.0 \%)$ & $3(15 \%)$ & \\
\hline Kidney & 39 & $26(53 \%)$ & $13(65 \%)$ & \multirow{5}{*}{0.48} \\
\hline Proximal ureter & 3 & $2(4.0 \%)$ & $1(5.0 \%)$ & \\
\hline Mid ureter & 6 & $4(8.0 \%)$ & $2(10 \%)$ & \\
\hline Distal ureter & 17 & $13(27 \%)$ & $4(20 \%)$ & \\
\hline Multiple & 4 & $4(8.0 \%)$ & $0(0.0 \%)$ & \\
\hline Hydronephrosis & 28 & 19 (39\%) & $9(45 \%)$ & \multirow{2}{*}{0.67} \\
\hline No hydronephrosis & 41 & 30 (61\%) & $11(55 \%)$ & \\
\hline Concomitant bladder $\mathrm{Ca}$ & 7 & $5(10 \%)$ & $2(10 \%)$ & \multirow{2}{*}{0.97} \\
\hline No bladder $\mathrm{Ca}$ & 62 & $44(90 \%)$ & $18(90 \%)$ & \\
\hline Urothelial Ca & 67 & 48 (98\%) & 19 (95\%) & \multirow{2}{*}{0.53} \\
\hline Benign lesion & 2 & $1(2.0 \%)$ & $1(5.0 \%)$ & \\
\hline Pathological stage $\geq \mathrm{T} 3$ & 31 & $21(44 \%)$ & $10(53 \%)$ & \multirow{2}{*}{0.51} \\
\hline$\leq \mathrm{T} 2$ & 36 & 27 (56\%) & $9(47 \%)$ & \\
\hline Grade 1 & 2 & $2(4.0 \%)$ & $0(0.0 \%)$ & \multirow{3}{*}{0.24} \\
\hline Grade 2 & 26 & 21 (44\%) & $5(26 \%)$ & \\
\hline Grade 3 & 39 & 25 (52\%) & 14 (74\%) & \\
\hline Lymph node involved & 2 & $1(2.0 \%)$ & $1(5.0 \%)$ & \multirow{2}{*}{0.53} \\
\hline No nodal involvement & 65 & 47 (98\%) & $18(95 \%)$ & \\
\hline Positive surgical margin & 12 & $8(17 \%)$ & $4(21 \%)$ & \multirow{2}{*}{0.67} \\
\hline Negative surgical margin & 55 & $40(83 \%)$ & 15 (79\%) & \\
\hline Mitomycin-C given & 18 & $11(23 \%)$ & $7(37 \%)$ & \multirow{2}{*}{0.27} \\
\hline No mitomycin C & 49 & 37 (77\%) & 12 (63\%) & \\
\hline Adjuvant chemo given & 10 & $4(8.3 \%)$ & 6 (31.6\%) & \multirow{2}{*}{$0.02^{*}$} \\
\hline No adjuvant chemo & 57 & $44(91.7 \%)$ & $13(68.4 \%)$ & \\
\hline
\end{tabular}

\section{TABLE 1: Perioperative characteristics of included patients}

*significant $p$-value

There was no change in the treatment strategy for any patient who underwent URS. One patient in each group had benign disease based on the histopathology report of the post-RNU specimen.

\section{Impact of URS on delay to definitive surgery}

There was a significant delay in RNU due to URS compared to the control group but none beyond the recommended 90-day target (Figure 1). The mean time \pm SD between the CTU and RNU was $86 \pm 37$ days 


\section{Cureus}

compared to $59 \pm 27$ in the control group ( $\mathrm{p}=0.007$ ). Seven patients were excluded from the delay analysis, as they had their surgery delayed for reasons unrelated to the URS findings.

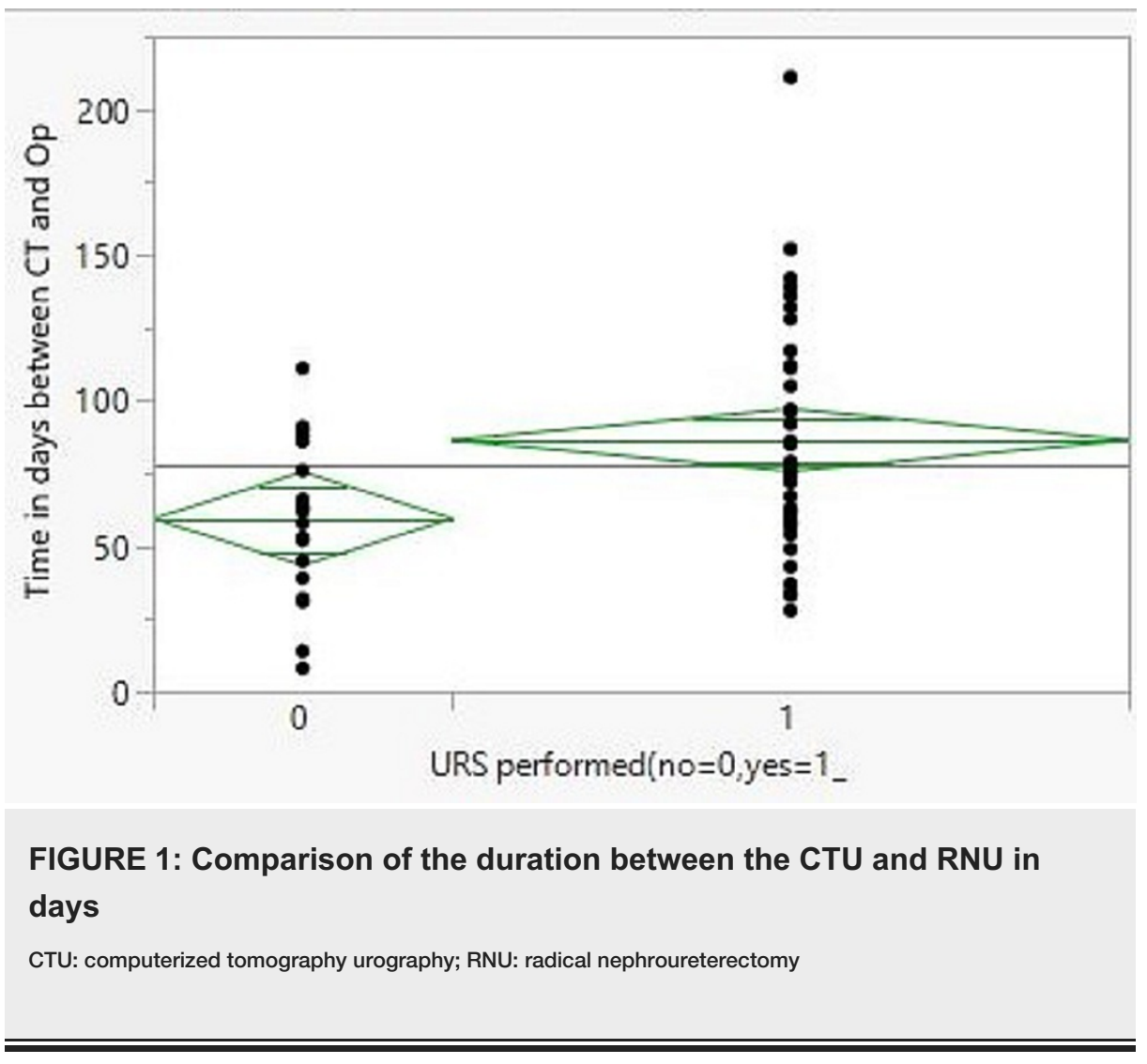

\section{Intravesical recurrence}

Fifty-nine (59) patients with a mean follow-up of 48.5 months were included in intravesical recurrence-free survival analysis ( 7 patients with concomitant bladder cancer, 2 patients with benign disease, and 1 patient who died from postoperative complication were excluded). The Kaplan-Meier curve (Figure 2) displayed better intravesical recurrence-free survival for the control group as compared to the URS group; however, the difference is insignificant (log-rank $\mathrm{p}$-value $=0.21$ ). The incidence of recurrence within the first 12 months postop was $28.2 \%$ in the URS group compared to $5.9 \%$ in the control group ( $\mathrm{p}=0.04)$. Overall recurrence in the URS group was $42.1 \%$ compared to $23.5 \%$ in the control group ( $\mathrm{p}=0.18$ ). 


\section{Cureus}

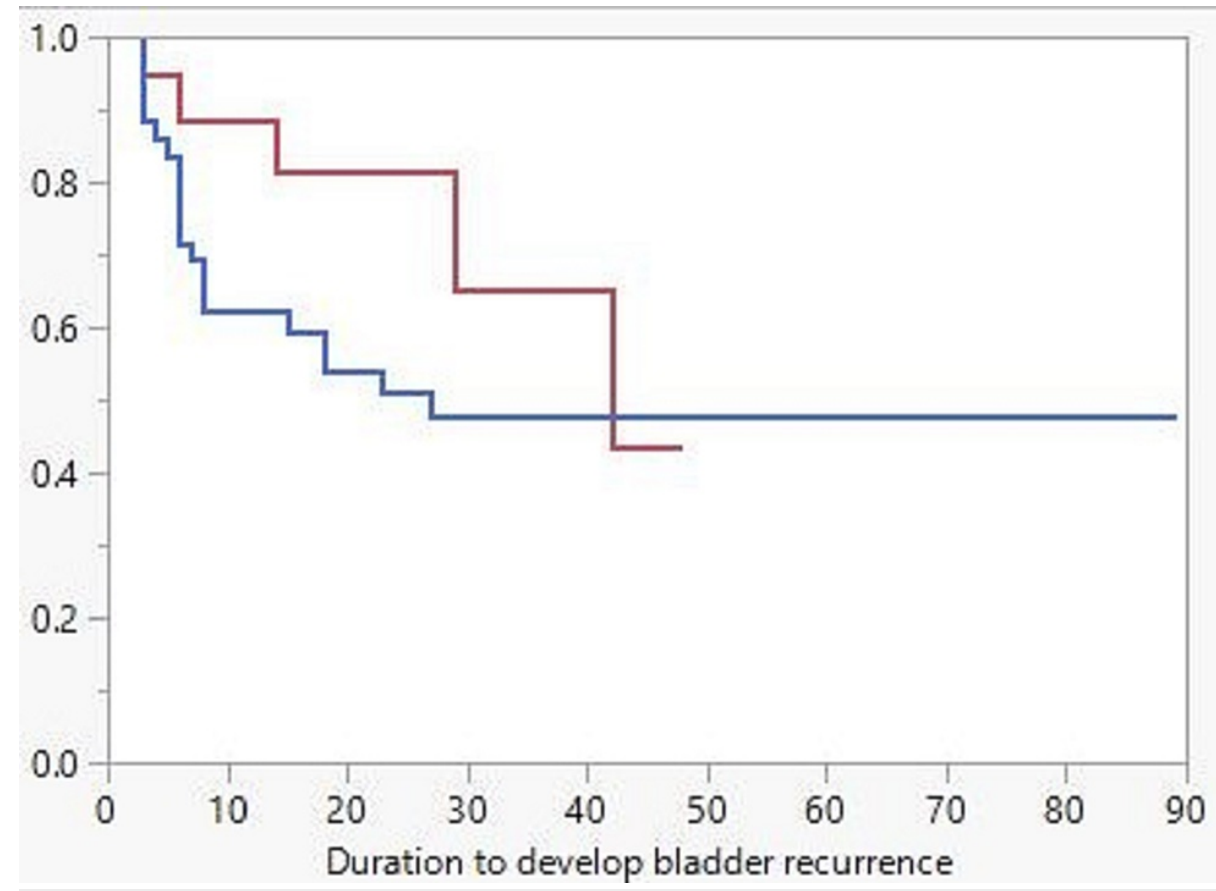

FIGURE 2: Kaplan-Meier curve for IVR-free survival; red line for the nonURS group and blue line for the URS group

IVR: intravesical recurrence; URS: ureteroscopy

All the available variables, including the presence of concomitant bladder urothelial carcinoma, were included in univariate and multivariate Cox regression analyses for intravesical recurrence (Table 2). In univariate analysis, concomitant bladder UC was significantly associated with a higher risk of recurrence (HR, 4.8; 95\%CI, 1.868-12.34; $\mathrm{p}=0.001$ ). In multivariate analysis, concomitant bladder UC was an independent risk factor for intravesical recurrence (HR, 15.01; 95\%CI, 3.311 - 68.07; p=0.0004). In addition, single-dose intravesical mitomycin-c was significantly associated with lower risk in multivariate analysis (HR 0.154; 95\%CI $0.025-0.922 ; \mathrm{p}=0.040$ ). 


\section{Cureus}

\begin{tabular}{|c|c|c|c|c|c|c|}
\hline \multirow{2}{*}{ Variable } & \multicolumn{3}{|c|}{ Univariate } & \multicolumn{3}{|c|}{ Multivariate } \\
\hline & $\mathrm{HR}$ & $95 \% \mathrm{Cl}$ & P value & $\mathrm{HR}$ & $95 \% \mathrm{Cl}$ & $P$ value \\
\hline Age & 0.984 & $0.943-1.029$ & 0.471 & 0.961 & $0.892-1.035$ & 0.299 \\
\hline Sex (Male vs Female) & 1.503 & $0.631-3.582$ & 0.357 & 2.694 & $0.763-9.514$ & 0.123 \\
\hline Stage ( $\geq T 3$ vs & 1.314 & $0.597-2.889$ & 0.497 & 0.456 & $0.093-2.219$ & 0.330 \\
\hline Grade (3 vs 1or2) & 1.549 & $0.699-3.429$ & 0.280 & 3.001 & $0.869-10.35$ & 0.082 \\
\hline Hydronephrosis & 0.802 & $0.357-1.802$ & 0.594 & 0.725 & $0.239-2.201$ & 0.570 \\
\hline Focality (Multi vs Uni) & 2.310 & $0.539-9.902$ & 0.259 & 3.217 & $0.551-18.76$ & 0.194 \\
\hline Delay of NU & 1.819 & $0.759-4.358$ & 0.179 & 0.777 & $0.234-2.571$ & 0.679 \\
\hline Concomitant BI UC & 4.800 & $1.868-12.34$ & $0.001^{x}$ & 15.01 & $3.311-68.07$ & $0.0004^{*}$ \\
\hline Diag. URS (yes vs no) & 1.809 & $0.681-4.802$ & 0.234 & 1.840 & $0.482-7.019$ & 0.372 \\
\hline Surgical margin & 1.916 & $0.715-5.129$ & 0.195 & 1.775 & $0.299-10.52$ & 0.527 \\
\hline Procedure (Lap vs open) & 2.358 & $0.319-1 / .41$ & 0.400 & 2.265 & $0.163-31.34$ & 0.541 \\
\hline Mitomycin C & 0.752 & $0.299-1.890$ & 0.544 & 0.154 & $0.025-0.922$ & $0.040^{*}$ \\
\hline Adjuvant Chemotherapy & 0.932 & $0.275-3.154$ & 0.910 & 1.256 & $0.174-9.044$ & 0.820 \\
\hline
\end{tabular}

TABLE 2: Cox regression model for risk factors of intravesical recurrence

*significant p-value

\section{Discussion}

Upper tract urothelial carcinoma is a rare, but challenging, form of urological cancer [8-9]. Assessment of the T stage by CTU is difficult and may underestimate the actual depth of underlying muscle involvement [10]. Studies have suggested more than $95 \%$ diagnostic accuracy for tumors that are obviously locally advanced, at least >pT3 tumors [11].

Diagnostic ureteroscopy may be performed to allow complete inspection of the collecting system and obtain a tissue biopsy. This may be performed for cases where the preoperative CT scan is unable to convincingly diagnose the presence of a urothelial carcinoma as the cause of a filling defect or collecting system mass. Furthermore, URS also lacks the ability of accurate staging and depths of invasion assessment. Diagnostic biopsies obtained are often small volume tissue samples that do not accurately represent the tumor [12].

This study displayed that, despite having a prior diagnostic ureteroscopy, all patients eventually underwent radical nephroureterectomy. There was a significant delay in performing RNU as compared to the patients who did not have URS; however, this was not beyond 90 days. Yet, comparing the two groups, there was no significant difference in the final tumor stage on histology.

One of the challenges in UTUC treatment is the risk of IVR that can affect up to $47 \%$ of patients [6]. A common concern with a prior diagnostic ureteroscopy is the incidence of increased IVR [6]. However, a few studies refute that risk [13]. This is postulated to occur due to tumor manipulation and the increased intrarenal pressures during ureteroscopy, which can lead to the shedding of cancer cells and implantation into the bladder [14]. This theory might explain our findings that the IVR was more obvious and significantly higher in the URS group during the first year post-RNU.

Our analysis for IVR included other characteristics such as gender, tumor location, upper tract tumor grade, unifocality or multifocality, and if there was any delay to definitive extirpative surgery. Surgical technique, i.e. open vs laparoscopic approach, also did not affect the rates of intravesical recurrence. Though not statistically significant, this finding is in line with multiple studies performed to investigate this, including the systemic analysis by Marchioni et al. in 2017, which found a significant incidence of intravesical recurrence following diagnostic ureteroscopy [6].

We found in our cohort of patients that the presence of concomitant bladder urothelial carcinoma was the single most predictive factor for intravesical recurrence, which is not unexpected. However, this was 
independent of the fact if the patient had a prior diagnostic ureteroscopy. Some authors have found a definite correlation between the presence of concomitant bladder urothelial carcinoma as a predictive factor for intravesical recurrence [15], whereas others did not find a significant correlation [16-17].

In our study, a single instillation of intravesical chemotherapy significantly reduced the incidence of intravesical recurrence. This underlies the principles of reducing circulating tumor cells and thus the implantation of tumor cells into the bladder, which was proven by a randomized controlled trial [18].

This is a retrospective review with all the limitations this implies. Notably, the selection of patients who will undergo URS and those who will not was undertaken on an individual basis prospectively by an individual urologist. This review may be subject to the risk of subtle bias with treatment selection for our comparison patient groups.

A further limitation of our study is the heterogeneity of URS techniques. There is variation in the use of semi-rigid or flexible scopes, the length of the procedure, and the use of access sheath. There is also variation in the use of perioperative ureteric stenting and the inherent impact of a stent towards disseminating tumor cells from a more proximal location to the bladder.

This study was designed to clarify the role of diagnostic URS. The current practice of reserving diagnostic URS only for cases that pose a diagnostic dilemma seems reasonable. In this study, given only two noncancer diagnoses from the overall cohort, which constitutes approximately $3 \%$ of patients, and with the anticipation that future research and investigative techniques will continue to improve, it may be reasonable to rely on CTU only for the majority of cases.

Only obtaining a definitive tissue diagnosis prior to RNU might not be a strong enough indication for URS when the patient can be assigned as high risk based on other factors such as multifocality and size of the lesion from the CTU. A fine balance needs to be struck between the possibility of benign lesion or misdiagnosis versus the increased risk of intravesical recurrence and potential delay to definitive extirpative surgery.

\section{Conclusions}

In our retrospective single-unit study, diagnostic ureteroscopy for CTU-detected upper tract urothelial carcinoma delayed definitive surgical treatment. Furthermore, it was associated with an increased risk of early intravesical recurrence in the URS group as compared to the control group. While URS can provide useful information and reassurance prior to major surgery, it must be used with caution in light of these findings.

\section{Additional Information \\ Disclosures}

Human subjects: Consent was obtained or waived by all participants in this study. Animal subjects: All authors have confirmed that this study did not involve animal subjects or tissue. Conflicts of interest: In compliance with the ICMJE uniform disclosure form, all authors declare the following: Payment/services info: All authors have declared that no financial support was received from any organization for the submitted work. Financial relationships: All authors have declared that they have no financial relationships at present or within the previous three years with any organizations that might have an interest in the submitted work. Other relationships: All authors have declared that there are no other relationships or activities that could appear to have influenced the submitted work.

\section{References}

1. Siegel R, Naishadham D, Jemal A: Cancer statistics, 2013. CA Cancer J Clin. 2013, 63:11-30. 10.3322/caac. 21166

2. Margulis V, Shariat SF, Matin SF, et al.: Outcomes of radical nephroureterectomy: a series from the Upper Tract Urothelial Carcinoma Collaboration. Cancer. 2009, 115:1224-33. 10.1002/cncr.24135

3. Rouprêt M, Babjuk M, Burger M, et al.: European Association of Urology Guidelines on upper urinary tract urothelial carcinoma: 2020 update. Eur Urol. 2021, 79:62-79. 10.1016/j.eururo.2020.05.042

4. Janisch F, Shariat SF, Baltzer P, et al.: Diagnostic performance of multidetector computed tomographic (MDCTU) in upper tract urothelial carcinoma (UTUC): a systematic review and meta-analysis. World J Urol. 2020, 38:1165-75. 10.1007/s00345-019-02875-8

5. Waldert M, Karakiewicz PI, Raman JD, et al.: A delay in radical nephroureterectomy can lead to upstaging . BJU Int. 2010, 105:812-7. 10.1111/j.1464-410X.2009.08821.x

6. Marchioni M, Primiceri G, Cindolo L, et al.: Impact of diagnostic ureteroscopy on intravesical recurrence in patients undergoing radical nephroureterectomy for upper tract urothelial cancer: a systematic review and meta-analysis. BJU Int. 2017, 120:313-9. 10.1111/bju.13935

7. Guo RQ, Hong P, Xiong GY, et al.: Impact of ureteroscopy before radical nephroureterectomy for upper tract urothelial carcinomas on oncological outcomes: a meta-analysis. BJU Int. 2018, 121:184-93.

10.1111/bju.14053 
8. Soria F, Shariat SF, Lerner SP, et al.: Epidemiology, diagnosis, preoperative evaluation and prognostic assessment of upper-tract urothelial carcinoma (UTUC). World J Urol. 2017, 35:379-87. 10.1007/s00345016-1928-x

9. Lughezzani G, Burger M, Margulis V, et al.: Prognostic factors in upper urinary tract urothelial carcinomas: a comprehensive review of the current literature. Eur Urol. 2012, 62:100-14. 10.1016/j.eururo.2012.02.030

10. Baard J, de Bruin DM, Zondervan PJ, Kamphuis G, de la Rosette J, Laguna MP: Diagnostic dilemmas in patients with upper tract urothelial carcinoma. Nat Rev Urol. 2017, 14:181-91.

11. Jinzaki M, Kikuchi E, Akita H, Sugiura H, Shinmoto H, Oya M: Role of computed tomography urography in the clinical evaluation of upper tract urothelial carcinoma. Int J Urol. 2016, 23:284-98. 10.1111/iju.13032

12. Rojas CP, Castle SM, Llanos CA, et al.: Low biopsy volume in ureteroscopy does not affect tumor biopsy grading in upper tract urothelial carcinoma. Urol Oncol. 2013, 31:1696-700. 10.1016/j.urolonc.2012.05.010

13. Lee HY, Yeh HC, Wu WJ, et al.: The diagnostic ureteroscopy before radical nephroureterectomy in upper urinary tract urothelial carcinoma is not associated with higher intravesical recurrence. World J Surg Oncol. 2018, 16:135. 10.1186/s12957-018-1411-9

14. Sankin A, Tin AL, Mano R, et al.: Impact of ureteroscopy before nephroureterectomy for upper tract urothelial carcinoma on oncologic outcomes. Urology. 2016, 94:148-53. 10.1016/j.urology.2016.05.039

15. Luo HL, Kang CH, Chen YT, Chuang YC, Lee WC, Cheng YT, Chiang PH: Diagnostic ureteroscopy independently correlates with intravesical recurrence after nephroureterectomy for upper urinary tract urothelial carcinoma. Ann Surg Oncol. 2013, 20:3121-6. 10.1245/s10434-013-3000-z

16. Ishikawa S, Abe T, Shinohara N, et al.: Impact of diagnostic ureteroscopy on intravesical recurrence and survival in patients with urothelial carcinoma of the upper urinary tract. J Urol. 2010, 184:883-7. 10.1016/j.juro.2010.05.027

17. Nison L, Rouprêt M, Bozzini G, et al.: The oncologic impact of a delay between diagnosis and radical nephroureterectomy due to diagnostic ureteroscopy in upper urinary tract urothelial carcinomas: results from a large collaborative database. World J Urol. 2013, 31:69-76. 10.1007/s00345-012-0959-1

18. Goel A, Paul S: Re: Tim O'Brien, Eleanor Ray, Rajinder Singh, Bola Coker, Ralph Beard, British Association of Urological Surgeons Section of Oncology. Prevention of bladder tumours after nephroureterectomy for primary upper urinary tract urothelial carcinoma: a prospective, multicentre, randomised clinical trial of a single postoperative intravesical dose of mitomycin C (the ODMIT-C Trial). Eur Urol 2011;60:703-10. Eur Urol. 2012, 61:e14; author reply e15. 10.1016/j.eururo.2011.11.037 